\title{
How to Become a Successful Pediatrician: An Inside Story of the Outside World
}

A newly passed postgraduate in Pediatrics, born after a long gestation of $8(5+3)$ years (in the protective womb of a medical college) is a novice to the outside world of practical pediatrics. He/she lacks the analytical skills and acumen of a private practitioner - to be able to judge and satisfy the demands of mothers bringing their children for check-ups. Initially, he/she thinks himself/herself as ultimate doctor deserving a daily practice of at least 50 patients, simply because he possesses the knowledge that a mutational analysis of the PLA2G6 gene is required for diagnosing Infantile Neuronal Axonal Dystrophy.

After few months, he/she realizes the eternal truth behind a lucrative practice is not what he/she was taught in those three years of extensive academic training but that there is much more to be learnt. These guidelines enlighten you with the secrets behind a flourishing practice - the deep rooted desire of any pediatrician.

1. Don't underestimate the power of CCF: Here CCF does not stand for congestive cardiac failure but is an eponym for cough, cold and diarrhea. The secret to conquer the hearts of innumerable mothers with their repetitive queries is to be well acquainted with as much general knowledge about these diseases as possible, and also throwing in some incomprehensible technical microbiological terms like Campylobacter jejuni, Shigella dysentery and Clostridium difficile. This will ensure satisfaction and you will be acknowledged as the most intelligent, knowledgeable and efficient pediatrician they have ever seen.

2. Recognize that the dictum in India is that every child is malnourished in the eyes of the mother. For example, if a child is $9 \mathrm{~kg}$ at 10 months and the mother is worried because the present weight gain no longer matches the gain she meticulously recorded in the initial months, smile (not sarcastically), take a deep breath (do not sigh) and explain how to enhance the feeding habits (not calories) rather than explaining the fundamental principles of growth curves. Here showing off your knowledge about age-appropriate weight criteria may not work!
3. Always be available on Whatsapp. This has 2 major advantages: First, creating an impression of being tech-savvy increases your credentials multifold. Second, parents can send you pictures of stool smeared nappies accompanied by details of color, consistency and amount of the stool with expressions of regret that technology cannot transmit smell invaluable to making a diagnosis.

4. Be extremely verbose: Pediatrics is more of psychological consultation of parents rather than medical consultation of the child. The more expressive and articulate you are the better your practice is. If you belong to the strong and silent category, there is a high risk of being rejected as 'unsatisfactory and egotist'. This can be easily rectified by joining coaching centers that teach you how to speak knowledgably at length on any topic, however irrelevant.

5. Ensure that the interiors of your consultation chamber are informative about your credentials. The walls should display ornately framed certificates of national and international conferences and CMEs attended since graduation days, with international certificates placed in most prominent areas. Adding the MBBS and MD degrees is optional. A display of conference mementos and impressive/Voluminous books (even if you have not read them once), in your chamber will attract more parents than actually reading them all!

The aforementioned guidelines are evidence-based in which extensive analysis of various case control studies (though with a methodology formulated by me) was undertaken. Anyone following these will definitely have a successful practice.

Competing interests: I do declare that I have a conflict of interest with primary interest of patient's welfare and secondary interest of falsely mesmerizing the patient's parents.

Funding: By all the patients who consulted me.

Puja Grover KaPoor Artemis Hospital, Sector 51, Gurgaon, Haryana, India. puja.kapoor79@gmail.com 